\title{
Relating Chromatographic Retention and Electrophoretic Mobility to the Ion Distribution Within Electrosprayed Droplets
}

\author{
C. Fredrik Bökman \\ Clinical Chemistry Laboratory, Falun Central Hospital, Falun, Sweden
}

Dan Bylund

Department of Natural Sciences, Mid Sweden University, Sundsvall, Sweden

\author{
Karin E. Markides and Per J. R. Sjöberg \\ Department of Analytical Chemistry, Uppsala University, Uppsala, Sweden
}

\begin{abstract}
Ions that are observed in a mass spectrum obtained with electrospray mass spectrometry can be assumed to originate preferentially from ions that have a high distribution to the surface of the charged droplets. In this study, a relation between chromatographic retention and electrophoretic mobility to the ion distribution (derived from measured signal intensities in mass spectra and electrospray current) within electrosprayed droplets for a series of tetraalkylammonium ions, ranging from tetramethyl to tetrapentyl, is presented. Chromatographic retention in a reversed-phase system was taken as a measure of the analyte's surface activity, which was found to have a large influence on the ion distribution within electrosprayed droplets. In addition, different transport mechanisms such as electrophoretic migration and diffusion can influence the surface partitioning coefficient. The viscosity of the solvent system is affected by the methanol content and will influence both diffusion and ion mobility. However, as diffusion and ion mobility are proportional to each other, we have, in this study, chosen to focus on the ion mobility parameter. It was found that the influence of ion mobility relative to surface activity on the droplet surface partitioning of analyte ions decreases with increasing methanol content. This effect is most probably coupled to the decrease in droplet size caused by the decreased surface tension at increasing methanol content. The same observation was made upon increasing the ionic strength of the solvent system, which is also known to give rise to a decreased initial droplet size. The observed effect of ionic strength on the droplet surface partitioning of analyte ions could also be explained by the fact that at higher ionic strength, a larger number of ions are initially closer to the droplet surface and, thus, the contribution of ionic transport from the bulk liquid to the liquid/air surface interface (jet and droplet surface), attributable to migration or diffusion will decrease. (J Am Soc Mass Spectrom 2006, 17, 318-324) (c) 2006 American Society for Mass Spectrometry
\end{abstract}

L arge efforts by the scientific community have been focused on gaining further understanding of electrospray as an ionization technique for mass spectrometry. Over the years, a number of equations have been developed that try to relate the observed mass spectrometric response at a given analyte and electrolyte concentration in the sprayed solution to a limited number of parameters. Tang and Kebarle [1] first used Iribarne and Thomson's ion evaporation theory [2] to develop a response model which initially utilized the rate constants for ion transfer from the

Published online January 25, 2006

Address reprint requests to Dr. P. J. R. Sjöberg, Department of Analytical Chemistry, Uppsala University, Box 599, SE-751 24 Uppsala, Sweden. E-mail: per.sjoberg@kemi.uu.se liquid surface of the charged droplet to the gas phase. In a later work [3], the model was modified slightly and surface activity was also included. Enke [4] developed a model that is based on equilibrium partitioning, for the analyte of interest, between the interior and the surface phases of a charged droplet. In one report from the Enke laboratory [5], the ESI response for small peptides was related to their chromatographic retention in a reversed-phase liquid chromatographic system. Higher ESI response was correlated $\left(R^{2}=0.882\right)$ with longer retention time in the system. In a paper by Zhou and Cook [6], a model was presented that qualitatively took account for the effects of ion pairing, surface activity, and electrophoretic mobility on the ESI response.

We have reported the development of a simplified method [7] for the determination of relative distribution 
coefficients $\left(K_{A} / K_{E}\right)$ for analyte and electrolyte ions, respectively, between the droplet surface and bulk solution, based on Enke's original model from 1997 [4]. Several experimental parameters that influence the results obtained with our proposed method have also been examined in more detail [8].

A central question for many applications is to find the degree to which the mass spectrum reflects the chemical environment in the condensed phase. A common assumption when relating an electrospray mass spectrum to the content of the analyzed solution is that the ions at, or close to, the droplet surface are those preferentially observed in the mass spectrum. In this study, we have continued our efforts to characterize our previously reported model system [7, 8], and focused on two parameters that can influence the ion distribution within the electrosprayed droplet, namely electrophoretic ion mobility and surface activity. Experimental data from both capillary electrophoresis (CE) and liquid chromatography (LC) were used to evaluate our previously obtained distribution coefficient values by efforts to combine the approach used in references [5] and [6]

\section{Theory}

A schematic of an electrosprayed droplet is shown in Figure 1. The droplet is hypothetically divided into two phases, a neutral interior phase and a charged surface phase. Eq 1, as derived by Enke [4], will express the partitioning of analyte and electrolyte ions between the interior (subscript $i$ ) and the surface phase (subscript $s$ ) of a droplet in a two component system. Under the assumption that the excess charge resides at the surface of the droplets (due to Coulombic repulsion), and that all other ions are paired with their counter ions, the ion equilibrium constants for the phase distribution can be expressed as a function of the concentrations of the ionic species present, i.e., analyte ion $\left(A^{+}\right)$, electrolyte ion $\left(E^{+}\right)$, and counter ion $\left(X^{-}\right)$.

$$
\frac{K_{A}}{K_{E}}=\frac{\left[E^{+} X^{-}\right]_{i}\left[A^{+}\right]_{S}}{\left[A^{+} X^{-}\right]_{i}\left[E^{+}\right]_{S}}
$$

When considering the excess charge, $Q$, which is the difference in the amount of cations and anions in the electrosprayed solution the equation was expressed as,

$$
\frac{K_{A}}{K_{E}}=\frac{\left[A^{+}\right]_{s}\left(C_{E}-\left[E^{+}\right]_{s}\right)}{\left(C_{A}-\left[A^{+}\right]_{s}\right)\left[E^{+}\right]_{s}}=\frac{\left[A^{+}\right]_{s}\left(C_{E}-[Q]+\left[A^{+}\right]_{s}\right)}{\left(C_{A}-\left[A^{+}\right]_{s}\right)\left([Q]-\left[A^{+}\right]_{s}\right)}
$$

where $C_{A}$ and $C_{E}$ are the total concentrations of analyte and electrolyte, respectively. In our laboratory, eq 3 [7] was developed for a simple determination of $K_{A} / K_{E}$.

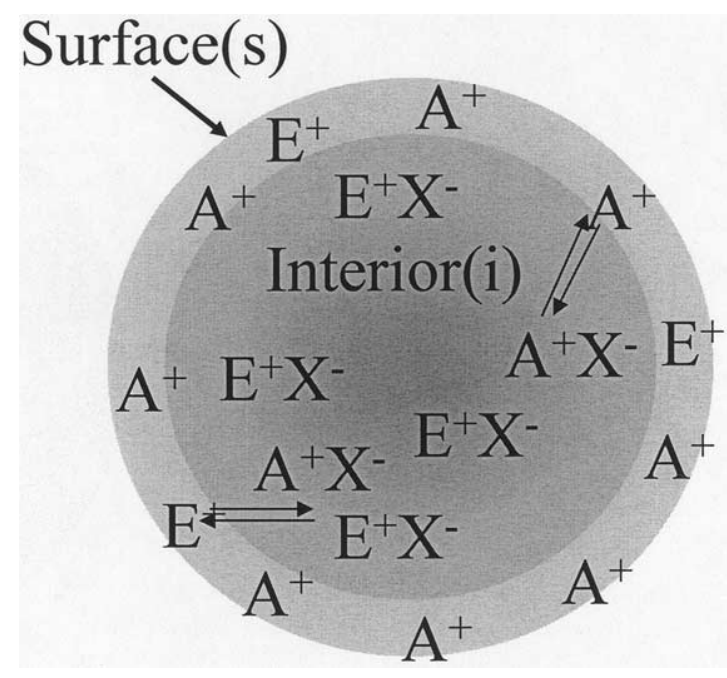

Figure 1. A schematic of an electrosprayed droplet. The droplet is hypothetically divided into two phases, a neutral interior phase and a charged surface phase. The $\left(\mathrm{A}^{+}\right),\left(\mathrm{E}^{+}\right)$, and $\left(\mathrm{X}^{-}\right)$represent the analyte, electrolyte, and counter ion, respectively.

$$
\frac{K_{A}}{K_{E}}=\frac{\left([Q]-[Q]^{0} \frac{R_{E}}{R_{E}^{0}}\right)\left(C_{E}-[Q]^{0} \frac{R_{E}}{R_{E}^{0}}\right)}{\left([Q]^{0} \frac{R_{E}}{R_{E}^{0}}\right)\left(C_{A}-[Q]+[Q]^{0} \frac{R_{E}}{R_{E}^{0}}\right)}
$$

This equation contains the analytical concentration of the analyte $\left(C_{A}\right)$ and the electrolyte $\left(C_{E}\right)$ together with two ESI-MS responses for the electrolyte, one when no analyte is present $\left(R_{\mathrm{E}}^{0}\right)$ and one when analyte is present $\left(R_{E}\right)$. Further, the equation contains two surface excess charge concentrations, one when no analyte is present $\left([Q]^{0}\right)$ and one when analyte is present $([Q])$.

Zhou and Cook [6] have examined in more detail several factors that could affect the surface partitioning coefficient. They developed an equation (eqn 4) for a two component system where ion pairing, surface activity, and ion mobility were considered.

$$
\frac{K_{A}}{K_{E}} \approx \frac{f_{A}(t)\left[K_{A, s a}+\frac{a \mu_{A} E t}{V_{s}(t)}\right]}{f_{E}(t)\left[K_{E, s a}+\frac{a \mu_{E} E t}{V_{s}(t)}\right]}
$$

In this equation, $f$ is the activity coefficient (approaching unity at infinite dilution), $K_{s a}$ is a surface activity constant, $a$ is the cross-sectional area of a droplet, $\mu$ is the ion mobility, $E$ is electric field strength, $t$ is the time, and $V_{s}$ the volume of the droplet surface. Cech et al. [5] considered $K_{A} / K_{E}$ as directly proportional to the retention factor $(k)$ of the analyte in reversedphase LC (reflecting hydrophobic interactions). If we instead make the assumption that the surface activity for an ion $\left(K_{i, s a}\right)$ is proportional to the retention factor of the analyte $\left(k_{i}\right)$ in reversed-phase LC according to eq 5 : 


$$
K_{i, s a}=\beta k_{i}
$$

where $\beta$ is a constant, then a combination of eqs 4 and 5 gives eq 6 :

$$
\begin{gathered}
\frac{K_{A}}{K_{E}} \approx \frac{f_{A}(t)}{f_{E}(t)} \cdot \frac{\beta k_{A}+\frac{a \mu_{A} E t}{V_{s}(t)}}{\beta k_{E}+\frac{a \mu_{E} E t}{V_{s}(t)}}=\frac{f_{A}(t)}{f_{E}(t)} \cdot \frac{k_{A}+\frac{\alpha(t)}{\beta} \mu_{A}}{k_{E}+\frac{\alpha(t)}{\beta} \mu_{E}} \\
=\chi(t) \cdot \frac{k_{A}+\delta(t) \mu_{A}}{k_{E}+\delta(t) \mu_{E}}
\end{gathered}
$$

which can be fitted to experimental LC and CE data for $k$ and $\mu$, respectively. In eq $6, \mathrm{X}$ is a coefficient that is dependent on the activity coefficient and $\delta$ is a coefficient describing the relative importance of surface activity and electrophoretic mobility (the higher the value of $\delta$, the larger the influence of ion mobility on the observed $K_{A} / K_{E}$ ). By evaluating the experimental data with eq 6 it is now possible to obtain knowledge about the relative importance of surface activity and ion mobility for the ion distribution within the electrosprayed droplet. This information can then be useful when the appearance of a mass spectrum should be related to the chemical environment of the sample in the condensed phase.

\section{Experimental}

\section{Sample and Solution Preparation}

The model compounds used in this study were bromide salts of tetramethylammonium (TMeABr), tetraethylammonium (TEtABr), tetrapropylammonium (TPrABr), tetrabutylammonium (TBuABr), and tetrapentylammonium (TPeABr), all from Fluka Chemie AG (Buchs, Switzerland). All chemicals were of at least $99 \%$ purity and used without further purification. Furthermore, deuterated tetramethylammoniumchloride (TMeACld12) was supplied by Cambridge Isotope Laboratories (Andover, MA). Stock solutions of the different tetraalkylammonium salts were prepared at $10 \mathrm{mM}$ in methanol (LC gradient grade) from Merck (Darmstadt, Germany). Sample solutions were prepared by diluting the stock solutions with different methanol/water mixtures to the desired concentration. Water was obtained from a Milli-Q plus purification system (Millipore Corporation, Bedford, MA). Hydrochloric acid, sodium chloride, and dimethyl sulfoxide (DMSO), all of p.a. (pro analysis) purity, were obtained from Merck.

\section{Determination of Retention Coefficient by Liquid Chromatography}

The chromatographic system consisted of an HPLC pump (PU-980, JASCO Co., Tokyo, Japan), a six port injection valve (C6W,Valco Instruments, Houston, TX) with a $20 \mu$ L loop and a Genesis C18 column $(150 \times 3.0$ $\mathrm{mm}, 4 \mu \mathrm{m}$ ) from Jones Chromatography (Hengoed, UK). The mobile phases contained $10 \mathrm{mM} \mathrm{HCl}$ and 100 $\mu \mathrm{M}$ TMeACl-d12 and varying amounts of methanol. A flow rate of $400 \mu \mathrm{L} / \mathrm{min}$ was used through the column, and split to $15 \mu \mathrm{L} / \mathrm{min}$ in a Valco tee before the pneumatically assisted electrospray interface. The sample consisted of $100 \mu \mathrm{M}$ TMeABr, TEtABr, TPrABr, $\mathrm{TBuABr}$ and $\mathrm{TPeABr}$ dissolved in water. Detection was made in the positive ion mode with a PE Sciex API-III+ triple quadrupole instrument (Concord, ON, Canada), equipped with an IonSpray interface. The fused silica spray capillary, $50 \mu \mathrm{m}$ i.d. and $187 \mu \mathrm{m}$ o.d., was centered in a stainless-steel capillary auxiliary assembly delivering $1.0 \mathrm{~L} / \mathrm{min}$ of air for pneumatically assisted ESI-MS. The spray tip was positioned about $20 \mathrm{~mm}$ from the interface plate, $\sim 10 \mathrm{~mm}$ off-axis and with a spray angle of $\sim 45^{\circ}$. The flow rate of dry nitrogen curtain gas, which was heated to $65^{\circ} \mathrm{C}$, was $1.4 \mathrm{~L} / \mathrm{min}$. The following mass spectrometer parameters were given set values: ion spray voltage (ISV) $4600 \mathrm{~V}$, interface plate voltage (IN) $650 \mathrm{~V}$, orifice lens (OR) $50 \mathrm{~V}$, and $\mathrm{AC}$ entrance rod (R0) $30 \mathrm{~V}$. Data were acquired in selected ion monitoring (SIM) mode with a dwell time of $50 \mathrm{~ms}$ using PE Sciex Tune software version 2.5-FPU.

\section{Determination of Ion Mobility by Capillary Electrophoresis}

The CE instrument was a home-built system that consisted of a high voltage power supply (Bertan, series 230, Hicksville, NY) with a platinum electrode placed in a small vial for the running electrolyte solution. Samples $(100 \mu \mathrm{M}$ each analyte and $1 \% \mathrm{vol} / \mathrm{vol}$ of dimethyl sulfoxide for monitoring the mobility of the electroosmotic flow) were introduced by hydrodynamic injection by elevating the sample vial $\sim 10 \mathrm{~cm}$ for $10 \mathrm{~s}$. An untreated fused silica capillary (Polymicro Technologies, Phoenix, AZ) with a length of $40 \mathrm{~cm}$ and $50 \mu \mathrm{m}$ i.d., $187 \mu \mathrm{m}$ o.d. were used for the separations. CE electrolyte solutions with different amounts of methanol $(0,30,50,70$, and 100\%, vol/vol) containing $10 \mathrm{mM}$ $\mathrm{HCl}$ and $10 \mathrm{mM} \mathrm{NaCl}$ were used. The effective potential over the CE capillary was $10.4 \mathrm{kV}$. Detection was made in the positive ion mode with a PE Sciex API-I single quadrupole instrument, equipped with an in-house assembled electrospray sheath-liquid interface. The high voltage power supply in the mass spectrometer was connected to ground through a $60 \mathrm{M} \Omega$ high voltage resistor [9]. The CE-separation capillary was centered in a stainless-steel capillary $200 \mu \mathrm{m}$ i.d., $400 \mu \mathrm{m}$ o.d. Other mass spectrometer parameters were similar to the parameters used in the LC-MS experiments. The spray assembly tip was positioned $20 \mathrm{~mm}$ from the interface plate, $\sim 5 \mathrm{~mm}$ off-axis, and aimed parallel with the ion optics. Make-up liquid, consisting of $70 \% \mathrm{vol} / \mathrm{vol}$ methanol containing $10 \mathrm{mM} \mathrm{HCl}$ and $\mathrm{NaCl}$, was delivered by a Harvard 22 syringe pump (Harvard Apparatus, SaintLaurent, Canada) at a flow-rate of $0.5 \mu \mathrm{L} / \mathrm{min}$. Between 


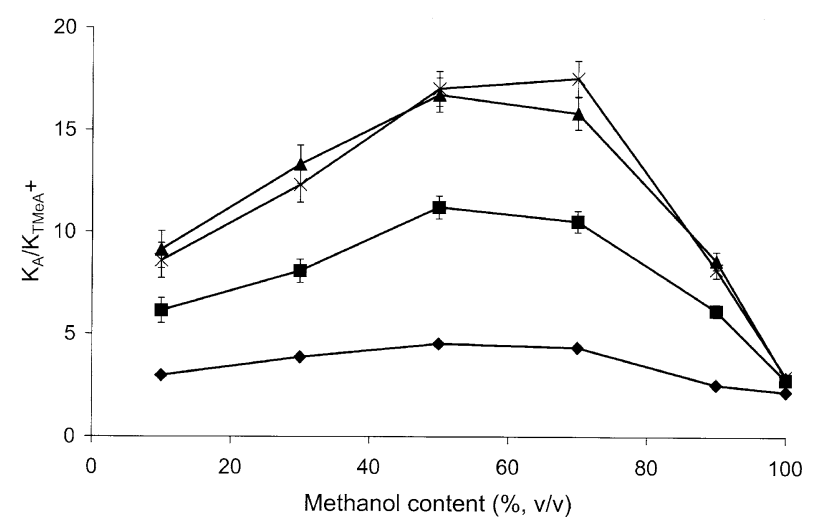

Figure 2. The droplet surface partitioning coefficient $\left(K_{A} / K_{E}\right)$ for different tetraalkylammonium compounds as a function of methanol content in the electrosprayed solution. The compounds are marked with the following symbols: filled diamond $\mathrm{TEtA}^{+}$, filled square $\mathrm{TPrA}^{+}$, filled triangle $\mathrm{TBuA}^{+}$, and the letter $\mathbf{X} \mathrm{TPeA}^{+}$. For some of the data points the standard deviation is smaller than the used symbol size.

each run, the capillary was flushed with fresh electrolyte solution. During the change of electrolyte in the CE capillary, the spray assembly was aimed with a large off-axis setting relative to the sampling orifice and a flow of nebulizer gas $(1.0 \mathrm{~L} / \mathrm{min}$ of air, zero grade, generated in house) was applied to remove excess liquid at the spray tip.

All mass spectral data, both in the CE- and LC-MS experiments, were recorded at unit mass resolution, and mass scale calibration was performed using a polypropylene glycol solution (PPG) supplied by the instrument manufacturer. At least duplicate injections were made in both CE- and LC-MS experiments. The chromatographic retention factors were not determined for $\mathrm{TBuA}^{+}$at $10 \% \mathrm{MeOH}$ and for $\mathrm{TPeA}^{+}$at 30 and $10 \%$ $\mathrm{MeOH}$ because of the very long retention times.

\section{Determinations of the Surface Partitioning Coefficient $\left(K_{A} / K_{E}\right)$ with Mass Spectrometry}

Determinations of the surface partitioning coefficient $\left(K_{A} / K_{E}\right)$ were made according to our previously reported method [7]. The method requires only two samples to be analyzed for each mobile phase composition. The first sample containing only a reference compound (the electrolyte) at a desired concentration, usually $0.2 \mathrm{mM}$, and the second sample containing similar amounts of electrolyte $\left(C_{E}\right)$ and analyte $\left(C_{A}\right)$ with a total concentration equal to the first sample (i.e., $0.2 \mathrm{mM}$ ). For the analysis of the samples a PE Sciex API-III+ triple quadrupole instrument, equipped with an IonSpray interface was used. The intensity for the electrolyte ion and the spray current were recorded for both samples and used in the calculation of $K_{A} / K_{E}$ (eqn 3). Mass spectrometer parameters were similar to the parameters used in the LC-MS experiments. For further details see reference [7].

\section{Results and Discussion}

\section{Effect of Organic Content}

In a previous study [8], we observed that when the methanol content of the electrosprayed solution was decreased below $50 \%$, the surface partitioning coefficient, determined by eq 3 , decreased for the model analytes. The observed changes of $K_{A} / K_{E}$, see Figure 2, are complex and require careful analysis of the system.

To investigate the behavior of the surface partitioning coefficient in more detail, we have chosen to describe the analyte/electrolyte system by retention factors $(k)$ and ion mobilities $(\mu)$ obtained from reversedphase LC and CE experiments, respectively [10]. The results from our determination of the retention factors for the model compounds are shown in Figure 3. A plot of $\log k$ versus organic modifier content in the mobile phase for a limited organic modifier range should give a straight line with a negative slope if only one type of interaction is present [11]. However, in our LC experiments, deviations from this theory were obtained for low retention values (data not shown). This could be because it is hard to accurately measure retention factors approaching zero. Another reason could be that the retention on the column was governed both by hydrophobic interactions with the C18 carbon chains, which we assume reflects the surface activity, and by some electrostatic interactions with residual silanol groups. To circumvent this problem, only experimental retention factors above 0.3 were considered to reflect the hydrophobic interactions of interest, and linear relationships between $\log k$ and the volume fraction of methanol in the mobile phase were then achieved. Both the intercept $\left(\log k_{w}\right)$ and the slope of these relationships were found to be linearly dependent on the number of carbon atoms in the analytes, and these dependencies were utilized to estimate $k$ for each analyte at all mobile phase compositions (Table 1).

Different transport mechanisms such as migration

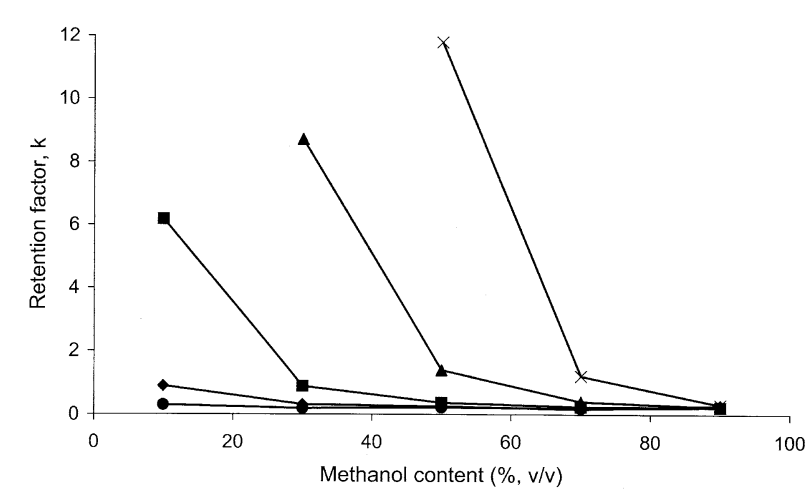

Figure 3. The retention factor $(k)$ for different tetraalkylammonium compounds as a function of methanol content in the mobile phase. The compounds are marked with the following symbols: filled circle $\mathrm{TMeA}^{+}$, filled diamond $\mathrm{TEtA}^{+}$, filled square $\mathrm{TPrA}^{+}$, filled triangle $\mathrm{TBuA}^{+}$, and the letter $\mathbf{X} \mathrm{TPeA}^{+}$. The standard deviation is smaller than the used symbol size. 
Table 1. Experimentally derived values for retention factor and electrophoretic mobility as a function of methanol content

\begin{tabular}{|c|c|c|c|c|c|c|c|c|c|c|}
\hline \multirow[b]{2}{*}{$\%$ Methanol } & \multicolumn{2}{|c|}{$\mathrm{TMeA}^{+}$} & \multicolumn{2}{|c|}{ TEtA $^{+}$} & \multicolumn{2}{|c|}{$\operatorname{TPrA}^{+}$} & \multicolumn{2}{|c|}{$\mathrm{TBuA}^{+}$} & \multicolumn{2}{|c|}{$\mathrm{TPeA}^{+}$} \\
\hline & k & $\mu^{\square}$ & k & $\mu^{\square}$ & k & $\mu^{\square}$ & k & $\mu^{\square}$ & k & $\mu^{\square}$ \\
\hline 10 & $7.4 e-2$ & 41.6 & $7.1 \mathrm{e}-1$ & 30.5 & $6.4 e+0$ & 20.9 & $7.2 e+1$ & 16.6 & $5.9 e+2$ & 13.9 \\
\hline 30 & $9.8 e-3$ & 32.0 & $9.4 e-2$ & 23.4 & $8.5 e-1$ & 16.2 & $9.6 e+0$ & 13.1 & $7.8 e+1$ & 11.2 \\
\hline 50 & $1.3 e-3$ & 21.6 & $1.2 e-2$ & 15.0 & $1.1 e-1$ & 9.5 & $1.3 e+0$ & 7.6 & $1.0 e+1$ & 6.5 \\
\hline 70 & $1.7 e-4$ & 21.0 & $1.6 e-3$ & 15.4 & $1.5 e-2$ & 9.7 & $1.7 e-1$ & 7.9 & $1.4 \mathrm{e}+0$ & 6.7 \\
\hline 90 & $2.3 e-5$ & 40.5 & $2.2 e-4$ & 35.2 & $2.0 e-3$ & 25.8 & $2.2 \mathrm{e}-2$ & 21.7 & $1.8 e-1$ & 18.8 \\
\hline 100 & $8.3 e-6$ & 61.1 & $7.9 e-5$ & 55.5 & $7.2 e-4$ & 42.6 & $8.1 e-3$ & 36.0 & $6.6 e-2$ & 31.4 \\
\hline
\end{tabular}

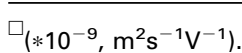

and diffusion can also influence the surface partitioning coefficient. During the spray formation the strong electric field that is present at the Taylor cone and liquid jet will probably alter the surface liquid composition in such a way that the abundance of those ions with higher mobilities will initially be increased at the droplet surface. Once the droplet has been formed the influence of the electric field on the ion distribution between the two phases is less certain. In the liquid droplet diffusion of the different species can play a role in the ion distribution. The relation between ion mobility and diffusion is expressed by the Einstein relation, $\mathrm{D}=$ $\mu \mathrm{kT} / \mathrm{ez}$ [12], where $\mathrm{k}$ is the Boltzmann constant, $\mathrm{T}$ is the absolute temperature, $\mathrm{e}$ is the charge of an electron, $\mathrm{z}$ is the charge number of the ion, $\mathrm{D}$ is the diffusion coefficient and $\mu$ is the ion mobility. Regardless of which the dominating transport mechanism is, diffusion and ion mobility are proportional, and we have in this study chosen to use the ion mobility parameter.

In a work by Zhou and Cook [6] an equation (eq 4) for a two component system was presented which expresses the surface partitioning coefficient as a function of ion pairing, surface activity and ion mobility. In this study we utilize a similar approach. The retention of our model compounds in the LC system was taken as a measure of hydrophobicity, which we also assume reflects the surface activity of the different compounds. In addition, the electrophoretic mobilities of our model compounds were determined using $\mathrm{CE}$, and the normalized mobilities at different methanol-water solvent compositions are shown in Figure 4. The shape of the different ion mobility curves resembles the inverse of the normalized solvent viscosity [13] curve, which is also shown in Figure 4. Plots of the inverse ion mobility in a given solvent versus ion mass gave straight lines with correlation coefficients better than 0.98 , indicating a rather good determination of the ion mobility in the solvent systems with minimal interaction with the silica surface of the CE capillary. Furthermore, the absolute value of the ion mobility obtained in this study for $100 \%$ water solutions correlates well with literature values [14].

According to the report by Cech et al. [5], an increase of the surface partitioning coefficient with decreasing organic modifier content would be expected. This is in contradiction with our results shown in Figure 2, where
$K_{A} / K_{E}$ for the model compounds decreases when the methanol content is decreased below $50 \%$. This observed behavior of the surface partitioning coefficients can be explained if the ion mobility is included in the model. The experimentally determined ion mobility (normalized values presented in Figure 4) and the derived ion mobility in Table 1 shows a minimum at $50-70 \%$ of methanol. This is in good agreement with the maximum viscosity of methanol/water mixtures at around 50\% methanol [13]. When the methanol content is decreased from 100 to $50 \%$, the viscosity increases [13], and both the mobilities and the differences in surface activities will work in the same direction (i.e., giving rise to higher $K_{A} / K_{E}$ values when the analyte has higher surface activity and lower mobility than the electrolyte). When the methanol content is further decreased below 50\% methanol, the viscosity starts to decrease and then mobility and surface activity will act in opposite directions regarding the influence on $K_{A} / K_{E}$.

When the data for retention factors and electrophoretic mobilities in Table 1 were fit to the surface partitioning coefficients shown in Figure 2, it was assumed that the activity coefficients were similar for the model compounds, i.e., $\mathrm{X}(t)$ was set to unity. The $\delta$-parameter could then be calculated by rearrangement of eq 6 according to

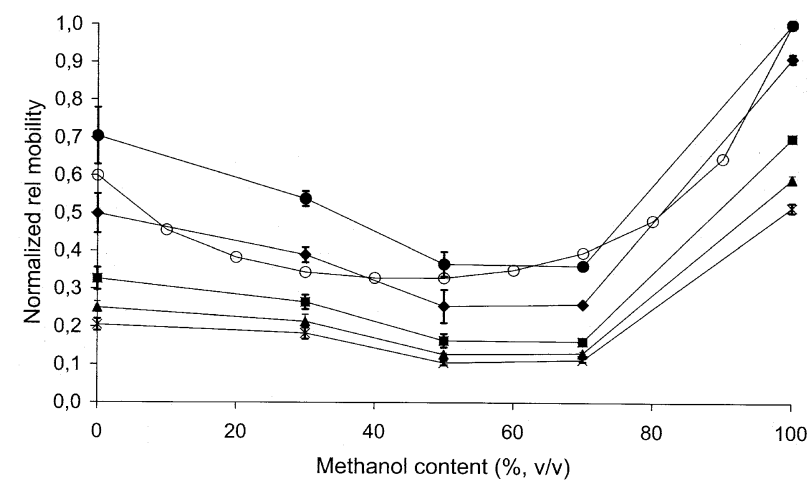

Figure 4. Normalized electrophoretic mobility for TAA ions versus solvent composition. The compounds are marked with the following symbols: filled circle $\mathrm{TMeA}^{+}$, filled diamond $\mathrm{TEtA}^{+}$, filled square $\mathrm{TPrA}^{+}$, filled triangle $\mathrm{TBuA}^{+}$, and the letter $\mathbf{X}$ $\mathrm{TPeA}^{+}$. The line with open circles represents the inverse of normalized solvent viscosity. For some of the data points the standard deviation is smaller than the used symbol size. 


$$
\delta=\frac{k_{A}-k_{E} K_{A} / K_{E}}{\mu_{E} K_{A} / K_{E}-\mu_{A}}
$$

The absolute value for the $\delta$-parameter, which describes the relative importance of surface activity and electrophoretic mobility, decreased with increasing methanol content, and it was also found to be compound-dependent as can be seen in Table 2. However, for each model compound the relative change of the $\delta$-parameter was similar when changing the methanol content.

Interpretation of the appearance of the $\delta$-parameter and its implication on any physical properties is not obvious. One physical parameter that could influence the $\delta$-parameter is the solvent surface tension. For methanol/water mixtures, the surface tension increases with increasing water content [15]. This could result in larger droplets at high water content even when nebulizer gas is used to assist droplet formation. When the generated droplets become larger, we assume that the importance of the mobility term, or diffusion, for the transport of analyte ions to the droplet surface increases. This agrees well with the observed increase in the $\delta$-parameter at higher water content in the electrosprayed solution (Table 2).

\section{Effect of Ionic Strength}

The data presented in Figure 2 were generated using a constant ionic strength of $0.2 \mathrm{mM}$. It has been observed that the instrumental response factor is influenced by the ionic strength $[8,16]$. Furthermore, it has also been reported that the droplet size is a function of ionic strength as measured by conductivity [17-20]. It was therefore of interest to investigate if also the surface partitioning coefficient is altered by a change in ionic strength and to determine the behavior of the corresponding $\delta$-parameter. As can be seen in Figure 5, ionic strength has a large influence on the surface partitioning coefficient. The $\delta$-parameter in this experiment was found to decrease by a factor of seven when the ionic strength was increased from $0.1 \mathrm{mM}$ to $0.2 \mathrm{mM}$ and decreased further by a factor of about two when the ionic strength was increased to $1 \mathrm{mM}$. This indicates that the importance of ionic transport to the droplet surface due to migration or diffusion decreases drasti-

Table 2. The $\delta$-parameter* obtained from eq 7 at different solvent composition for the different model analytes

\begin{tabular}{rrrrr}
\hline \% Methanol & TEtA $^{+}$ & TPrA $^{+}$ & TBuA $^{+}$ & TPeA $^{+}$ \\
\hline \hline 10 & $5.2 e-3$ & $2.5 e-2$ & $2.0 e-1$ & $1.7 e+0$ \\
30 & $5.6 e-4$ & $3.2 e-3$ & $2.3 e-2$ & $2.0 e-1$ \\
50 & $8.0 e-5$ & $4.2 e-4$ & $3.5 e-3$ & $2.9 e-2$ \\
70 & $1.2 e-5$ & $6.2 e-5$ & $5.1 e-4$ & $3.8 e-3$ \\
90 & $2.4 e-6$ & $8.2 e-6$ & $6.7 e-5$ & $5.8 e-4$ \\
100 & $7.9 e-7$ & $5.5 e-6$ & $5.8 e-5$ & $4.4 e-4$ \\
\hline
\end{tabular}

$*\left(\mathrm{Vsm}^{-2}\right)$.

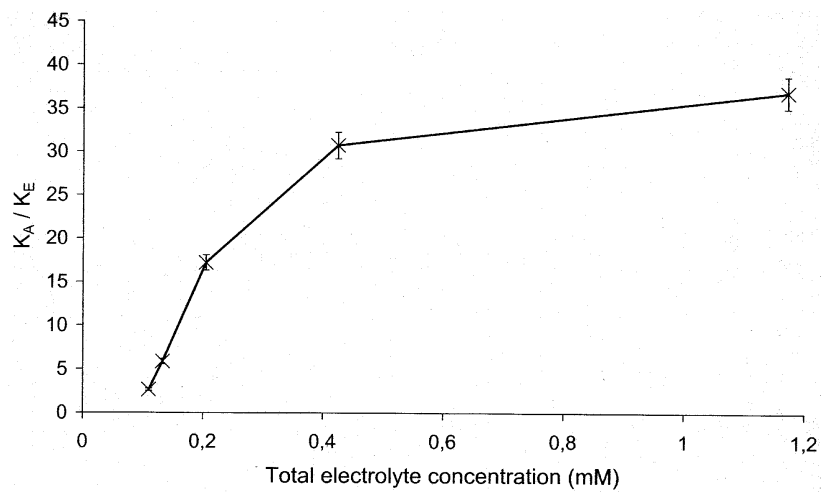

Figure 5. The droplet surface partitioning coefficient $\left(K_{A} / K_{E}\right)$ for tetrapentylammonium as a function of ionic strength. Solvent contains $50 \%$ methanol. For some of the data points the standard deviation is smaller than the used symbol size.

cally at low ionic strength levels. An explanation for the observed effect could be that, at higher concentrations, a larger number of ions are closer to the droplet surface creating a more compact electrical double layer [16] so that the importance of ionic transport to the droplet surface decreases. Results, which indicate that the droplet size influence the surface partitioning coefficient, have previously been reported [8]. An increase in $K_{A} / K_{E}$ was observed upon increasing the nebulizer gas flow above $0.6 \mathrm{~L} / \mathrm{min}$. We made the assumption that the shear force on the liquid jet emerging from the spray capillary increases with increased nebulizer gas flow [21] and, thus, generates a larger number of droplets with initially smaller diameter. The smaller droplets that are formed in this way will have an inherent increased number of ions from the bulk solution that will come closer to the liquid-air interface surface.

To further explore the effects of ionic strength on the surface partitioning coefficient, two sets of experiments were performed. The solvent composition was altered between 10 and $90 \%$ methanol at two different ionic strength levels $(0.15 \mathrm{mM}$ and $1.5 \mathrm{mM})$. The difference in the $K_{A} / K_{E}$ behavior between the two ionic strength levels for the $\mathrm{TPeA}^{+}$ion, shown in Figure 6, could once again be explained based on the relative importance of ion transport to the droplet surface due to migration or diffusion and surface activity. It can be assumed that at a higher ionic strength level the importance of migration or diffusion for the transport of analyte ions to the surface will decrease. This assumption is supported by the fact that lower values for the $\delta$-parameters are obtained for the higher ionic strength level.

\section{Conclusions}

Further insights in how the mass spectral response relate to the actual composition of the analyzed sample is important in many applications. In cases when complex samples are to be studied, matrix effects are often encountered. A separation step is often necessary in which the solvent composition may change from that of 


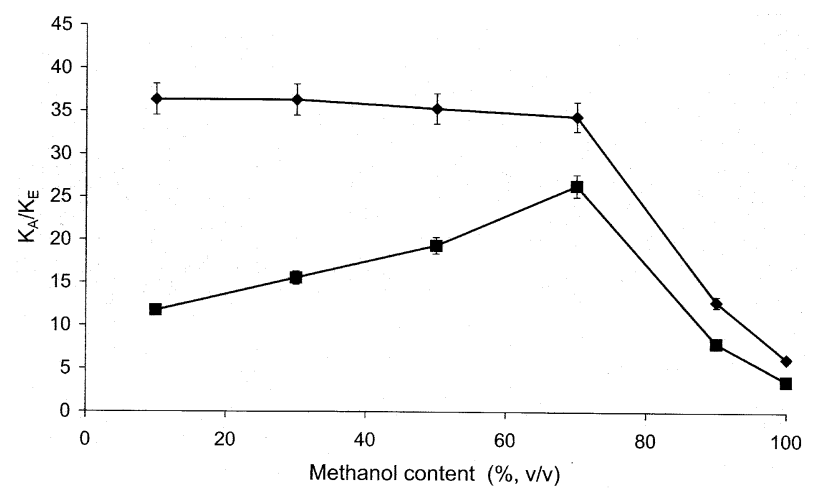

Figure 6. The droplet surface partitioning coefficient $\left(K_{A} / K_{E}\right)$ for tetrapentylammonium as a function of solvent composition at two different ionic strengths, filled square $0.15 \mathrm{mM}$ and filled diamond $1.5 \mathrm{mM}$. For some of the data points the standard deviation is smaller than the used symbol size.

the original sample. In this study, we have investigated the effect of analyte hydrophobicity and electrophoretic mobility on the partitioning of the analyte/electrolyte ions to the electrosprayed droplet surface. By altering the solvent composition, it was found that the influence of ion mobility on the droplet surface partitioning decreases with increasing organic content. A physical effect of the increased organic content is a decrease in surface tension, which we assume will give rise to a decrease in size of the generated droplets. As the size of a droplet decreases over its lifetime, we expect that the importance of the ion mobility for the transport of ions to the surface will subsequently become smaller. This agrees well with the observed behavior of the $\delta$-parameter, which in our model reflects the importance of ion mobility relative to surface activity. The same observation was made upon increasing the ionic strength of the solvent system, which is known to give rise to a decrease in droplet size but at the same time increase the ion density at the droplet surface.

We believe that the results presented in this paper can serve as a guideline to develop strategies to control the appearance of the mass spectrum, i.e., controlling discrimination effects or relative intensities between ions. When complex samples are to be analyzed, selection of a solvent system that provides high viscosity, i.e., low mobility and diffusion, will be beneficial for the relative intensity of surface-active compounds compared with more polar hydrophilic compounds with high mobility. Thus, if the relative intensity of polar hydrophilic compounds is to be enhanced, a selection of a solvent system with low viscosity should be beneficial.

With the results presented in this paper in mind, we also believe that the question often raised regarding how the mass spectrum reflects the chemical environment in the condensed phase can be answered in a more careful way. However, precautions should be taken when results for proteolytic compounds and larger biomolecules are considered, as they differ considerably from the model compounds used in this study.

\section{Acknowledgments}

The authors gratefully acknowledge financial support from the Swedish Natural Research Council, project K-5104-706 and from the Swedish Foundation for Strategic Research. They also thank Applied Biosystem, Sweden and AstraZeneca R and D Mölndal, Sweden for their generous gifts of the API I and API III+ instruments.

\section{References}

1. Tang, L.; Kebarle, P. Effect of the Conductivity of the Electrosprayed Solution on the Electrospray Current. Factors Determining Analyte Sensitivity in Electrospray Mass Spectrometry. Anal. Chem. 1991, 63, 2709-2715.

2. Iribarne, J. V.; Thomson, B. A. On the Evaporation of Small Ions from Charged Droplets. J. Chem. Phys. 1976, 64, 2287-2294.

3. Tang, L.; Kebarle, P. Dependence of Ion Intensity in Electrospray Mass Spectrometry on the Concentration of the Analytes in the Electrosprayed Solution. Anal. Chem. 1993, 65, 3654-3668.

4. Enke, C. G. A Predictive Model for Matrix and Analyte Effects in Electrospray Ionization of Singly-Charged Ionic Analytes. Anal. Chem. 1997, 69, 4885-4893.

5. Cech, N. B.; Krone, J. R.; Enke, C. G. Predicting Electrospray Response from Chromatographic Retention Times. Anal. Chem. 2001, 73, 208-213.

6. Zhou, S.; Cook, K. D. A. Mechanistic Study of Electrospray Mass Spectrometry: Charge Gradients Within Electrosprayed Droplets and Their Influence on Ion Response. J. Am. Soc. Mass Spectrom. 2001, 12, 206-214.

7. Sjöberg, P. J. R.; Bökman, C. F.; Bylund, D.; Markides, K. E. A Simple Method for Determination of Ion Distribution Within Electrospray Droplets. Anal. Chem. 2001, 73, 23-28.

8. Sjöberg, P. J. R.; Bökman, C. F.; Bylund, D.; Markides, K. E. Factors Influencing the Determination of Analyte Ion Surface Partitioning Coefficients in Electrosprayed Droplets. J. Am. Soc. Mass Spectrom. 2001, 12, 1001-1010.

9. Bruins, A. P. 1997; Cole, R. B., Ed.; In Electrospray Ionization Mass Spectrometry, Fundamentals, Instrumentation and Applications; pp 107-136.Wiley-Interscience: New York,

10. Harris, D. C. In Quantitative Chemical Analysis; W. H. Freeman and Company: New York, 2002.

11. Baczek, T.; Markuszewski, M.; Kaliszan, R.; van Straten, M. A. Claessens, H. A. Linear and Quadratic Relationships Between Retention and Organic Modifier Content in Eluent in Reversed Phase High-Performance Liquid Chromatography: A Systematic Comparative Statistical Study. J. High Resolut. Chromatogr. 2000, 23, 667-676.

12. Atkins, P. W. In Physical Chemistry; Oxford University Press: Oxford, 1986.

13. Snyder, L. R.; Kirkland, J. J.; Glajch, J. L. In Practical HPLC Method Development; John Wiley and Son, Inc.: New York, 1997; p 726.

14. Pospichehal, J.; Gebauer, P.; Bocek, P. Measurement of Mobilities and Dissociation Constants by Capillary Isotachophoresis. Chem. Rev. 1989, $89,419-430$.

15. Cech, N. B.; Enke, C. G. Effect of Affinity for Droplet Surface on the Fraction of Analyte Molecules Charged During Electrospray Droplet Fission. Anal. Chem. 2001, 73, 4632-4639.

16. Constantopoulos, T. L.; Jackson, G. S.; Enke, C. G. Effect of Salt Concentration on Analyte Response Using Electrospray Ionization Mass Spectrometry. J. Am. Soc. Mass Spectrom. 1999, 10, 625-634.

17. Smith, D. P. H. The Electrohydrodynamic Atomization of Liquids. IEEE Trans. Ind. Appl. 1986, IA-22, 527-535.

18. Fernandez de la Mora, J.; Loscertales, I. G. The Current Emitted by Highly Conducting Taylor Cones. J. Fluid Mech. 1994, 260, 155-184.

19. Rosell-Llompart, J.; Fernandez de la Mora, J. Generation of Monodisperse Droplets 0.3 to $4 \mathrm{um}$ in Diameter from Electrified Cone-Jets of Highly Conducting and Viscous Liquids. J. Aerosol Sci. 1994, 25, 1093-1119.

20. Loscertales I. G.; Fernandez de la Mora, J. Experiments on the Kinetics of Field Evaporation of Small Ions from Droplets. J. Chem. Phys. 1995, 103, 5041-5059

21. Kim, J. H.; Nakajima, T. Aerodynamic Influences on Droplet Atomization in an Electrostatic Spray. JSME Int. J. Series B 1999, 42, 224-229. 